\section{(A) Check for updates}

Cite this: Nanoscale, 2020, 12, 9647

\title{
A novel split mode TFBAR device for quantitative measurements of prostate specific antigen in a small sample of whole blood $\dagger$
}

\author{
Ewelina Wajs, (D) * $t^{\mathrm{a}}$ Girish Rughoobur, (D) ${ }^{\mathrm{b}}$ Keith Burling, ${ }^{\mathrm{c}}$ Anne George, ${ }^{\mathrm{d}}$ \\ Andrew J. Flewitt (iD $\S^{a}$ and Vincent J. Gnanapragasam $\S^{c, d, e, f}$
}

\begin{abstract}
Easy monitoring of prostate specific antigen (PSA) directly from blood samples would present a significant improvement as compared to conventional diagnostic methods. In this work, a split mode thin film bulk acoustic resonator (TFBAR) device was employed for the first time for label-free measurements of PSA concentrations in the whole blood and without sample pre-treatment. The surface of the sensor was covalently modified with anti-PSA antibodies and demonstrated a very high sensitivity of $101 \mathrm{kHz} \mathrm{mL} \mathrm{ng}{ }^{-1}$ and low limit of detection (LOD) of $0.34 \mathrm{ng} \mathrm{mL}^{-1}$ in model spiked solutions. It has previously been widely believed that significant pre-processing of blood samples would be required for TFBAR biosensors. Importantly, this work demonstrates that this is not the case, and TFBAR technology provides a costeffective means for point-of-care (POC) diagnostics and monitoring of PSA in hospitals and in doctors' offices. Additionally, the accuracy of the developed biosensor, with respect to a commercial auto analyser (Beckman Coulter Access), was evaluated to analyse clinical samples, giving well-matched results between the two methods, thus showing a practical application in quantitative monitoring of PSA levels in the whole blood with very good signal recovery.
\end{abstract}

Received 15th January 2020 Accepted 10th April 2020

DOI: $10.1039 / \mathrm{d} 0 \mathrm{nr} 00416 \mathrm{~b}$ rsc.li/nanoscale the UK and over 11000 men die because of the disease. This makes prostate cancer the second most common cause of cancer-related death for men in the UK, where one in eight men will develop prostate cancer in their lifetime and approximately 400000 men are now living with the disease. Additionally, the NPCA's latest report also suggests that the number of men with locally advanced disease and their potential "under-treatment" have increased, as compared to previous years. ${ }^{2}$ Therefore, like in diabetes management, encouraging men to self-test for prostate specific antigen (PSA) using a point-of-care (POC) testing kits is emerging as a significant health care need. This is particularly important for many men in whom the cancer is likely to be indolent but requires surveillance or regular monitoring rather than immediate treatment. $^{3}$

Prostate cancer is one of the few cancers with a known biomarker used for screening, diagnosis and monitoring after treatment. ${ }^{4}$ PSA is a $34 \mathrm{kDa}$ glycoprotein expressed predominantly by the prostate gland and present in large amounts in its tissue and semen. Although, PSA is not prostate specific and it can be present in other body fluids and tissues in healthy men as well as in women, it is still a very important biomarker in prostate cancer diagnostics. ${ }^{5}$ In female ejaculate PSA levels are almost as high as in male, but its high concentrations can further be found in breast milk and amniotic 
fluid. PSA is also present in the serum of women with breast, lung, or uterine cancer and in some patients with renal cancer. Thus, PSA as a single biomarker cannot be always directly linked to prostate cancer only, but undoubtedly testing for its elevated levels in the blood $\left(>4 \mathrm{ng} \mathrm{mL}^{-1}\right)$ can increase the chances for finding cancer in its earliest, most curable stage. ${ }^{6}$ Most importantly, by monitoring levels of PSA in the blood indicates how well cancer treatment has worked and it helps significantly in further cancer treatment and prognosis. Typically, PSA should drop to very low levels after surgery or radiation treatment for prostate cancer. ${ }^{7}$ Despite PSA testing being already widely available, it still largely relies on sample processing in clinical laboratories, which makes it impractical and too expensive for many applications. Thus, the need for a POC self-testing system has led to constant development of novel detection strategies that are suitable for miniaturisation as well as easy, rapid and accurate PSA measurements. Many different types of detection systems for PSA analysis from serum or blood samples have been previously reported in the literature, i.e. optical, electrochemical or mass-based methods. ${ }^{8-13}$ However, these are yet to be implemented in a regular POC test for rapid and inexpensive monitoring of PSA levels that is clinically accepted. They also tend to give PSA ranges rather than absolute values and are more used for detection of high levels rather than give granularity on actual measure levels. This is crucial if the disease is to be monitored accurately. Some commercially available PSA testing kits include home screening tests e.g. the SELFCheck ${ }^{\circledR}$ or PRIMA®, and lab testing kits, such as ELISA. However, most hospital laboratories measure total PSA by 2-site immunoassay on large automated analysers (Siemens Centaur, Beckman Coulter Access, Roche Elecsys, etc.). An important issue is how well these tests work in the context of a POC or patient administered test i.e. measurements made using whole human blood from a pin prick sample.

TFBAR gravimetric sensors can recognise biological species by detecting a very small change in mass attached to their sensing surface. A general structure of this device is presented in the Fig. 1; the device fabrication and operation was described in detail elsewhere. ${ }^{14}$ The essence of the device is that two resonant frequencies are produced in a single structure. The first resonator (R1) with an anti-PSA antibody modified gold surface responds to the biomolecule (antigen) attachment, whilst the second resonator (R2) without the gold layer is not sensitive to it. Yet, they both respond to any other influences (e.g. temperature, humidity). This means that the difference between these two resonant frequencies $\Delta\left(f_{2}-f_{1}\right)$ can be used to quantitatively measure the target analyte specifically from a sample. Key features of TFBAR devices include their very small size (the sensing area is typically $\sim 100 \times 100 \mu \mathrm{m}$ ), low power consumption, ease of multiplexing on a single, small chip and low cost. ${ }^{15}$ These characteristics make TFBAR devices very attractive for POC immunotesting. ${ }^{16}$

In general, there are many difficulties that various sensing systems still need to overcome, when using a fresh whole
(A)

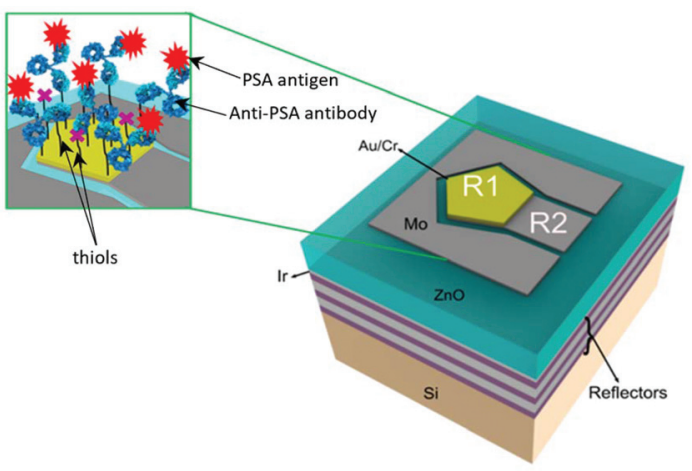

(B)

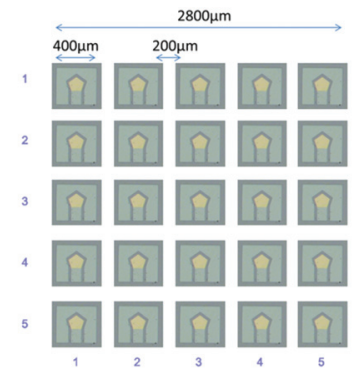

Fig. 1 (A) A schematic of the split-mode TFBAR biosensor with the functional gold layer only on a pentagonal section of the molybdenum top electrode, illustrating the regions corresponding to the first (R1/f $\left.f_{1}\right)$ and second $\left(R 2 / f_{2}\right)$ resonances; the enlargement shows the sensing surface (thiol + blocking + anti-PSA antibody) and target (PSA antigen) detection. (B) Optical image of $5 \times 5$ chip array of the TFBAR devices.

blood samples for testing, even the existing commercial finger prick tests for blood glucose monitoring in diabetics. Whole blood has one of the most complex matrices as compared to other body fluids, containing many various matrix components that can easily affect response of bioanalytical processes. Most notably, measurements performed on real samples from patients often create major difficulties in sample handling as well as data interpretation due to patient/ to/patient variability and a multitude of interfering analytes that need to be either removed or ignored by a selective enough sensor. There are often major sample preparation steps involved in removing interfering entities from the sample, such as red and white blood cells or lipids. ${ }^{17-20}$ Therefore, it has widely been believed that non-specific surface interactions would mean that also TFBAR devices would require complex fluids, like blood, to be pre-processed for successful biosensing. This would be rendered impractical for POC testing and it would result in increased associated costs. In this work, a previously reported acoustic split mode resonator has been used for the first time for direct measurements of PSA levels in whole blood from patients in a hospital setting. To demonstrate a proof-of-concept for this novel detection method based on TFBAR technology, a simple bioassay for total PSA detection was used. This type of biosensor demonstrated high sensitivity and low LOD and most importantly, it was done without sample pre-processing. Results were compared with those from the commercial Beckman Coulter Access analyser. 


\section{Experimental}

All reagents used in this work are described in the ESI. $\dagger$

\section{Biofunctionalisation of the TFBAR devices and total PSA measurements}

The formation of self-assembly monolayer (SAM) of alkanethiol on the gold layer on the TFBAR devices was done by immersing them in a freshly prepared (5 mM) 11-mercaptoundecanoic acid for $24 \mathrm{~h}$ at $4{ }^{\circ} \mathrm{C}$. Next, the modified surface was extensively washed with ethanol to remove any unbound molecules. The formation of SAMs was followed by additional surface-blocking step using 6-mercapto-1-hexanol (0.1 M) for $1 \mathrm{~h}$ at room temperature (RT). For antibody coupling, treatment of water-soluble carbodiimide and succinimide compounds was performed immediately by immersing the TFBAR devices in a freshly prepared solution of EDC (0.2 M) and NHS (50 $\mathrm{mM}$ ) for $30 \mathrm{~min}$, what activated the carboxylic acid end groups on thiol molecules. Anti-PSA polyclonal antibodies were covalently linked to the activated SAMs by spotting with $10 \mu \mathrm{L}$ of a $0.5 \mathrm{mg} \mathrm{mL}^{-1}$ solution for $1 \mathrm{~h}$ at RT. The remaining active ester sites were blocked with $1.0 \mathrm{M}$ ethanolamine $(\mathrm{pH}$ 8.5) for $30 \mathrm{~min}$ at RT. The surface optimisation related to the concentrations of the thiol and capture antibody is described in the ESI (Fig. S2 $\dagger$ ). Functionalised TFBAR devices were subsequently used for the PSA antigen detection from buffered and whole blood samples.

For the TFBAR detection system, a small volume $(10 \mu \mathrm{L})$ of either PSA in buffered solution or a fresh blood sample (from 7 patients) was spotted on the antibody-modified gold active surface and left to incubate for $5 \mathrm{~min}$ and $15 \mathrm{~min}$ in the humidity chamber, respectively (section 2.2, ESI $\dagger$ ). These allowed the recognition of the target protein by the immobilised receptors. All samples were taken as part of an ethically approved study (Ethics 03/018, DIAMOND national study) in our centre with informed consent obtained from all participants. All experiments were performed in compliance with relevant laws or guidelines of Cambridge University and approved by the NRES Committee East of England, UK. After extensive rinsing with MilliQ water and drying with nitrogen, the devices were measured using $150 \mu \mathrm{m}$ pitch ground-signalground (GSG) probes connected to a network analyser (NA-E5062A, Keysight Technologies, Santa Rosa, CA, USA), see sections 1 and 2, ESI. $\dagger$ To assess the reproducibility, the measurements were repeated 5 times for each sample concentration. The devices were cleaned with an argon plasma using a rf (13.56 MHz) Reactive Ion Etching (RIE) tool and checked for its reusability (Fig. S3, ESI $\dagger$ ).

Total PSA measurements with commercial chemiluminescent immunoassay (Beckman Coulter Access)

For the Access assay, the sample was added to a reaction vessel containing a mouse monoclonal anti-PSA antibody conjugated to alkaline phosphatase and paramagnetic particles coated with a second mouse monoclonal anti-PSA antibody. After incubation, materials bound to the paramagnetic particles were held in a magnetic field while other unbound materials were washed away. A chemiluminescent substrate (Lumi Phos 530) was added to the vessel. Light was generated by reaction with the PSA-alkaline phosphatase conjugate. The intensity of the light generated is proportional to the PSA concentration in the sample. The PSA concentration in the sample was then determined from a factory-generated stored multi-point calibration curve. Calibration is traceable to WHO Reference Preparation 96/670.

\section{Results and discussion}

\section{Gravimetric performance and characterisation of TFBAR biosensor using spiked solutions}

The potential of the split mode TFBAR biosensor as a platform for developing a sensitive and specific assay for PSA early detection is demonstrated below. Fig. 2A shows an example of the TFBAR biosensor response to the attachment of the (1) thiol (11-MUA) + blocking (6-mercapto-1-hexanol), (2) anti-PSA antibody and (3) PSA antigen. This response was monitored by measuring the real part of the electrical admittance $(\mathrm{Y})$ in the frequency range from $0.5 \mathrm{GHz}$ to $3.0 \mathrm{GHz}$. As can be seen, when each new layer of molecules is attached to the active

(A)

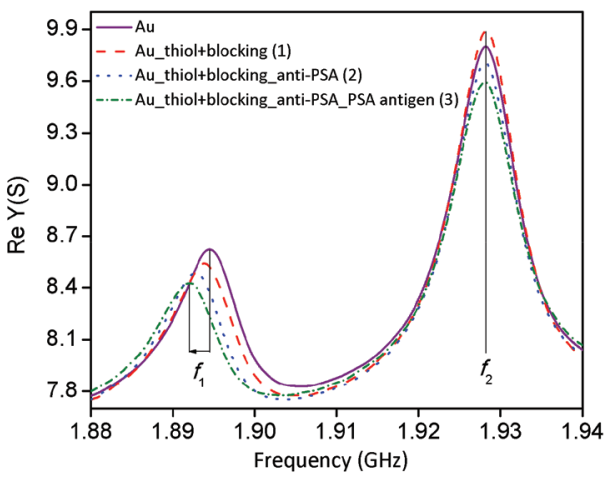

(B)

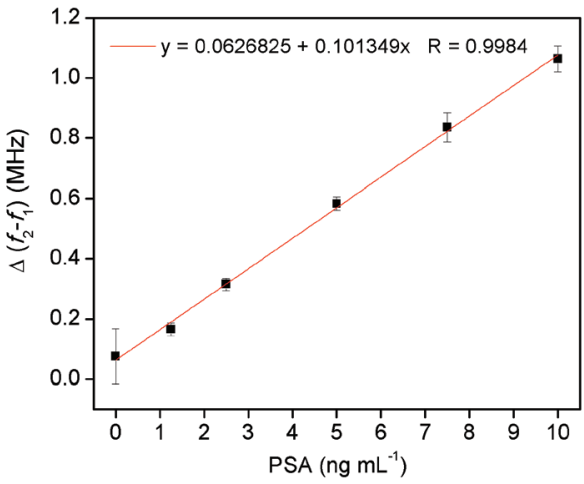

Fig. 2 (A) An increase in the difference between the two resonances $f_{2}-f_{1}$ observed in a split mode TFBAR biosensor due to the attachment of subsequent molecules onto $\mathrm{Au}$ active surface: (1) thiol + blocking; (2) thiol + blocking + anti-PSA; (3) thiol + blocking + anti-PSA + PSA antigen. (B) Corresponding calibration curve $y=0.063+0.1 x ; R=$ 0.9984 . 
surface of the TFBAR biosensor, the distance between the two frequencies $\Delta\left(f_{2}-f_{1}\right)$ increases. By measuring these changes while increasing the concentration of the analyte (PSA antigen), we obtained a calibration plot (see Fig. 2B), see also sections 5 and 6 in the ESI. $\uparrow$ The calibration plot showed excellent linearity $(R 2=0.9984)$ and the limit of detection (LOD) was found to be $0.34 \mathrm{ng} \mathrm{mL}{ }^{-1}$, calculated as 3 times the standard deviation of the $y$-intercept of the regression line divided by the slope. At the dynamic range observed in Fig. 2B, the assay lies in the clinically relevant range of PSA with label-free and rapid detection. Sensitivity of the assay reported from the slope of the calibration curve was $101 \mathrm{kHz} \mathrm{mL} \mathrm{ng}{ }^{-1}$, highlighting the effectiveness of the developed acoustic sensor in the screening of this important biomarker. The reproducibility of the TFBAR devices for quantifying PSA was investigated over the entire linear range and data showed that the relative standard deviation (RSD) was less than $12 \%$, for five independent experiments (from 1 to $10 \mathrm{ng} \mathrm{mL}^{-1}$ ). Selectivity and specificity of the TFBAR biosensor is crucial to its successful application for a direct blood screening. It was confirmed by performing measurements in the absence of the anti-PSA antibody in both buffered and whole blood samples. As demonstrated in Fig. S4 in the ESI, $\uparrow$ no response was observed. This showed high specificity of the anti-PSA modified surface to the PSA antigen and excellent surface coverage against other non-specific interactions. This was further confirmed by performing measurements in the presence of human serum albumin, which is the most abundant protein in human blood (section 4, ESI $\dagger$ ). The recovery of PSA in this case was found to be 104\% and 106\% in buffered and whole blood samples, respectively. Thus, further confirming that the TFBAR biosensors are selective and specific for the detection of PSA antigen.

The great advantages of the TFBAR technology over other conventional methods are: (i) it is simpler and less costly compared to ELISA-type assays (i.e. Access immunoassay), (ii) it does not require additional labelling for the detection, (iii) it can be performed in a single reaction without additional reagents and (iv) it is compact and therefore, portable.

\section{TFBAR application for the detection of PSA from clinical samples}

One of the main limitations of most conventional methodologies involving biological samples is the need to include pretreatment steps in order to minimise possible matrix interference effects. Herein, the target is to allow a direct sample analysis, without any sample pre-treatment. Thus, the developed split-mode TFBAR biosensor was used in the gravimetric detection of the total PSA in the whole blood samples from patients in the hospital. Fresh samples were taken and divided into aliquots, one for the Access immunoassay and one for the TFBAR detection.

The TFBAR measurements $\left(\Delta\left(f_{2}-f_{1}\right)\right)$ were performed using a portable network analyser (FieldFox, Model: N9913A from Keysight Technologies) and the unknown concentrations of PSA antigen in the whole blood samples were determined using the calibration plot obtained from the buffered solutions (Fig. 2B). The results acquired from both methods (TFBAR
Table 1 PSA levels measured in EDTA whole blood samples in $\mathrm{ng} \mathrm{mL}^{-1}$ with the developed TFBAR biosensor and standard Access immunoassay

\begin{tabular}{|c|c|c|c|}
\hline $\begin{array}{l}\text { Sample } \\
\text { from } \\
\text { patients }\end{array}$ & $\begin{array}{l}\text { TFBAR } \\
\text { biosensor } \\
\left(\mathrm{ng} \mathrm{mL} \mathrm{L}^{-1} \pm \mathrm{SD}\right)\end{array}$ & $\begin{array}{l}\text { TFBAR } \\
\text { biosensor } \\
\text { (\% RSD) }\end{array}$ & $\begin{array}{l}\text { Access } \\
\text { immunoassay } \\
\left(\mathrm{ng} \mathrm{mL}^{-1}\right)\end{array}$ \\
\hline 1 & $1.81 \pm 0.86$ & 47.5 & 0.47 \\
\hline 2 & $4.20 \pm 0.35$ & 8.3 & 3.25 \\
\hline 3 & $4.50 \pm 0.70$ & 15.5 & 3.90 \\
\hline 4 & $6.00 \pm 0.86$ & 14.3 & 5.29 \\
\hline 5 & $6.30 \pm 0.51$ & 8.0 & 5.70 \\
\hline 6 & $6.40 \pm 0.82$ & 12.8 & 5.73 \\
\hline 7 & $9.15 \pm 0.42$ & 4.5 & 9.60 \\
\hline
\end{tabular}

detection and commercial Access immunoassay) are summarised in the Table 1.

The obtained values of PSA concentration in blood were in majority higher from TFBAR sensor than those obtained by Access immunoassay. Also, the reproducibility of the TFBAR responses compared to those from buffered solutions showed higher RSD values (up to $15.5 \%$ ) for five independent experiments (from 1 to $10 \mathrm{ng} \mathrm{mL}{ }^{-1}$ ). At concentrations below $1 \mathrm{ng}$ $\mathrm{mL}^{-1}$ the TFBAR sensor showed very high $\mathrm{RSD}$ of $47.5 \%$, however this does not have high clinical impact as in general,

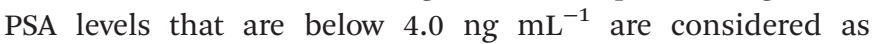
normal. Furthermore, these differences in the measurements may be due to insufficient blocking of the surface of the TFBAR sensor to prevent all non-specific interactions from a complex matrix such as whole blood and/or insufficient washing cycles of the device after the last step of incubation with the whole blood samples. Additionally, the use of polyclonal antibodies for capture could increase false positives due to sample contamination.

It may be possible to achieve even higher performance of the TFBAR sensor through further optimisation of its surface and immobilisation techniques. Our recent findings indicate that the sensitivity of the split mode TFBAR sensor depends on the thickness and roughness of the vacuum-deposited gold film. However, it also depends on the uniformity of the immobilisation layer and cleanliness of the sensing surface prior to functionalisation. ${ }^{21,22}$ It should be noted that whilst high sensitivity of $101 \mathrm{kHz} \mathrm{mL} \mathrm{ng}{ }^{-1}$ was achieved for PSA measurements in buffered solutions, the sensitivity in actual human whole blood samples decreased to $80 \mathrm{kHz} \mathrm{mL} \mathrm{ng}{ }^{-1}$. This could be due to the fact that analyte detection in a complex matrix such as blood is extremely difficult. Since blood contains thousands of various competing biomolecules and many of them at much higher concentrations than the target analyte, there is a high probability of false positives due to binding of the capture antibody to a non-target molecule that has a similar structural motif.

The possible effect of the serum matrix on target detection using the TFBAR biosensor was also studied by spiking blood samples with different amounts of PSA antigen (Table S1, $\left.\operatorname{ESI}^{\dagger}\right)$. As the signal recovery was close to $100 \%$ it indicated the applicability of the system to the analysis of a real clinical samples. 


\section{Accuracy validation of the developed TFBAR biosensor with commercial Access immunoassay}

The best way to compare the performance and effectiveness of the two analytical methods is to show their correlation and to determine their level of agreement. A correlation plot between the TFBAR biosensor and Access immunoassay is presented in Fig. 3A. The slope value of 0.81 (from a theoretical value of 1) indicates a good positive correlation between the PSA levels obtained by both methods. However, even though two methods have good correlation, it does not necessarily mean that they are in a good agreement. Therefore, we used the Bland-Altman method ${ }^{23}$ to assess the agreement between these two techniques. In Fig. 3B, the difference between Access immunoassay and TFBAR results (the bias) are plotted against the average of the two measurements. The agreement between these two quantitative measurements can be evaluated by constructing limits of agreement. The limits of agreement were calculated by using the mean and standard deviation (SD) of the differences between two measurements (mean bias \pm 1.96 times its SD). Furthermore, the Bland-Altman method recommends that $95 \%$ of the data points should lie within the calculated limits of agreement. As can be seen in Fig. 3B, the average of

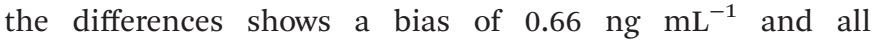
measurements lie within the area defined by the calculated

(A)

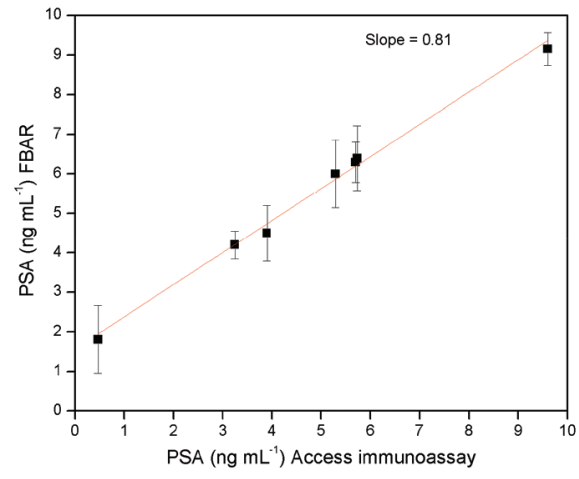

(B)

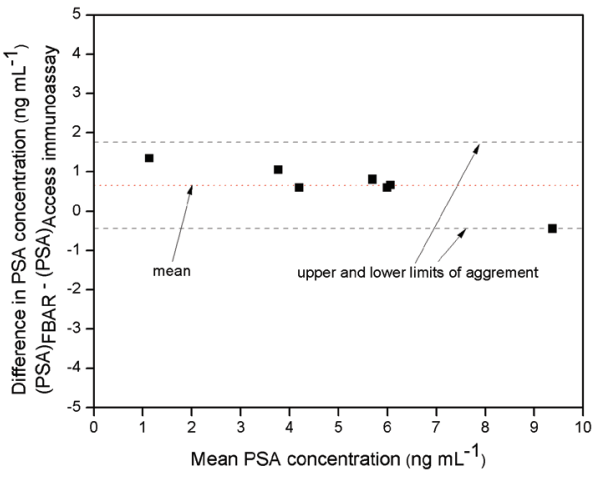

Fig. 3 (A) Correlation between PSA levels measured in a whole blood samples using the developed TFBAR biosensor and commercial access immunoassay results. (B) Bland-Altman plot comparing the results of the gravimetric measurements with Access immunoassay results. limits of agreement - which are clinically relevant. Therefore, a good agreement between the TFBAR sensor and commercial immunoassay was obtained.

Consequently, more work is needed to develop an integrated microfluidic system, which can further improve the performance of the TFBAR biosensor. Making this system fully automated with embedded electronics can minimise or even eliminate the "human error" during the experiment. The development of additional receptors and immobilisation methods for multiplexed assays could also help to improve the detection reproducibility of the TFBAR device. However, it is notable that the difference in TFBAR readings and Access immunoassay is in the sub $1 \mathrm{ng} \mathrm{mL}^{-1}$ range which is a very acceptable error margin in the context of utility in clinical practice. Particularly so, as PSA readings are known to fluctuate with normal biological variations. ${ }^{24}$ Alternatively, a standard correction can be applied to TFBAR reading, and so a future POC test could have an error of $1 \mathrm{ng} \mathrm{mL}^{-1}$ built in its system.

\section{Conclusions}

A split mode TFBAR biosensor offers distinct advantages over conventional methods for PSA monitoring, including a simple and label-free PSA detection with fewer manipulative steps, which speeds up the whole process. A low detection limit of $0.34 \mathrm{ng} \mathrm{mL}{ }^{-1}$ was achieved with a very high sensitivity of $101 \mathrm{kHz} \mathrm{mL} \mathrm{ng}{ }^{-1}$. This highly reproducible sensor platform was easily adapted for gravimetric detection of clinically relevant PSA levels in very small volumes of blood $(10 \mu \mathrm{L})$ and without sample pre-treatment. The capability to detect PSA directly from the complex matrix such as whole blood containing hundreds of thousands of competing proteins show a tremendous advantage over conventional detection techniques. This sensor platform could be easily multiplexed for a detection of a panel of PSA isoforms for even more effective patient screening and large patient sample analysis towards early detection of prostate cancer. ${ }^{25}$ The measurements can be done rapidly, at a very low cost and in a fully portable and automated format - a POC relevance. Furthermore, since the turnaround times for TFBAR sensing are much shorter, substantial savings are possible in diagnostic workup schedules. Ultimately, all those advantages, including the application to early cancer detection and disease monitoring, will have significant implications in reducing the cost to healthcare providers whilst improving the quality of care to patients. Efforts are currently underway to develop a multiplexed biomarker array with integrated microfluidic system for a real time, fast analysis.

\section{Conflicts of interest}

AJF is a Director of Sorex Sensors Ltd, which is commercialising FBAR sensors. 


\section{Acknowledgements}

The authors gratefully acknowledge the support of this work through a Medical Research Council Confidence in Concept award, CRUK Cambridge Cancer Early Detection Pump Priming Grant. The authors acknowledge infrastructure support from the Cambridge Biomedical Research Campus and from the Cancer Research UK Cambridge Centre.

\section{Notes and references}

1 Cancer Research UK [WWW Document], n.d., URL: https:// www.cancerresearchuk.org/health-professional/cancer-statistics/statistics-by-cancer-type/prostate-cancer (accessed 6.5.19).

2 National Prostate Cancer Audit [WWW Document], n.d., URL: https://www.npca.org.uk/(accessed 6.5.19).

3 S. W. D. Merriel, L. Hetherington, A. Seggie, J. T. Castle, W. Cross, M. J. Roobol, V. Gnanapragasam, C. M. Moore, M. Ashworth, E. Bradley, K. Cass, P. Cornford, J. Keanie, S. Little, K. Mastris, A. Nairn, J. Oxley, C. Parker, A. Patel, R. Porter, L. Powell, J. Richenberg, M. Roland, M. Varma, D. Victor and C. Waymont, BJU Int., 2019, 124, 47.

4 B. Bohunicky and S. A. Mousa, Nanotechnol., Sci. Appl., 2010, 4, 1 .

5 A. Fragoso and C. K. O'Sullivan, Electrochemical Immunosensor for Detection of Proteic Cancer Markers, in Biosensors and Molecular Technologies for Cancer Diagnostics, ed. K. E. R. A. Herold, CRC Press, Taylor \& Francis Group, 2012, p. 596.

6 S. A. Brosman, 2015, [WWW Document], URL: https:// emedicine.medscape.com/article/457394-overview\#section CharacteristicsofProstateSpecificAntigen (accessed 9.18.19).

7 M. Fox, [WWW Document]. Jan. 12, 2017, n.d., URL: https://emedicine.medscape.com/article/457394-overview\# section CharacteristicsofProstateSpecificAntigen (accessed 9.18.19).

8 I. E. Tothill, Semin. Cell Dev. Biol., 2009, 20, 55.
9 G. Wu, R. H. Datar, K. M. Hansen, T. Thundat, R. J. Cote and A. Majumdar, Nat. Biotechnol., 2001, 19, 856.

10 M. Kuriyama, S. Akimoto, H. Akaza, Y. Arai, M. Usami, K. Imai, Y. Tanaka, H. Yamazaki, Y. Kawada, K. Koiso, O. Yoshida, T. Kotake, H. Yamanaka, T. Machida, Y. Aso and J. Shimazaki, Jpn. J. Clin. Oncol., 1992, 22, 393.

11 H. Adel Ahmed and H. M. E. Azzazy, Biosens. Bioelectron., 2013, 49, 478.

12 A. I. Barbosa, A. P. Castanheira, A. D. Edwards and N. M. Reis, Lab Chip, 2014, 14, 2918.

13 K. Jung, R. Zachow, M. Lein, B. Brux, P. Sinha, K. Jung, S. Lenk, D. Schnorr and S. A. Loening, Urology, 1999, 53, 155.

14 E. Wajs, G. Rughoobur and A. J. Flewitt, Nanoscale, 2018, 10(30), 14395.

15 A. J. Flewitt, J. K. Luo, Y. Q. Fu, L. Garcia-Gancedo, X. Y. Du, J. R. Lu, X. B. Zhao, E. Iborra, M. Ramos and W. I. Milne, J. Non-Newtonian Fluid Mech., 2015, 222, 209.

16 P. Von Lode, Clin. Biochem., 2005, 38, 591.

17 M. L. Chiu, W. Lawi, S. T. Snyder, P. K. Wong, J. C. Liao and V. Gau, JALA, 2010, 15, 233.

18 S. R. Corrie, J. W. Coffey, J. Islam, K. A. Markeyd and M. A. F. Kendalla, Analyst, 2015, 140, 4350.

19 K. Wiener, [WWW Document], 2003, URL: https:// acutecaretesting.org, (accessed 3.14.20).

20 N. Bhalla, P. Jolly, N. Formisano and P. Estrela, Essays Biochem., 2016, 60, 1.

21 V. Tabard-cossa, M. Godin, I. J. Burgess, T. Monga and R. B. Lennox, Anal. Chem., 2007, 79, 8136.

22 R. Datar, S. Kim, S. Jeon, P. Hesketh, S. Manalis, A. Boisen and T. Thundat, MRS Bull., 2009, 34, 449.

23 J. M. Bland and D. G. Altman, Lancet, 1986, 1, 307.

24 J. A. Eastham, E. Riedel, P. T. Scardino, M. Shike, M. Fleisher, E. Lanza, L. Latkany and C. B. Begg, J. Am. Med. Assoc., 2003, 289(20), 2695.

25 V. J. Gnanapragasam, K. Burling, A. George, S. Stearn, A. Warren, T. Barrett and B. Koo, Sci. Rep., 2016, 6, 35364. 